\title{
Discrete Weighted Pseudo Almost Automorphic Solutions of Nonautonomous Difference Equations
}

\author{
Xiaoqing Wen ${ }^{1}$ and Hongwei Yin ${ }^{1,2}$ \\ ${ }^{1}$ School of Sciences, Nanchang University, Nanchang 330031, China \\ ${ }^{2}$ Numerical Simulation and High-Performance Computing Laboratory, School of Sciences, Nanchang University, \\ Nanchang 330031, China
}

Correspondence should be addressed to Hongwei Yin; hongwei-yin@hotmail.com

Received 6 December 2015; Accepted 24 March 2016

Academic Editor: Silvia Romanelli

Copyright (C) 2016 X. Wen and H. Yin. This is an open access article distributed under the Creative Commons Attribution License, which permits unrestricted use, distribution, and reproduction in any medium, provided the original work is properly cited.

We introduce the concept of a discrete weighted pseudo almost automorphic function and prove some basic results. Further, we investigate the nonautonomous linear and semilinear difference equations and obtain the weighted pseudo almost automorphic solutions of both these kinds of difference equations, respectively. Our results generalize the ones by Lizama and Mesquita (2013).

\section{Introduction}

The almost automorphic functions that were introduced initially by Bochner [1] are significant and related to some aspects of differential geometry. From then on, the almost automorphic solutions for deterministic differential systems were extensively investigated [2-5]. One can refer to the monograph [6]. Later, the concept of the pseudo almost automorphic function was proposed by N'Guérékata in [6], which seems to be more generalization of the pseudo almost periodic function $[7,8]$. In 2009, Blot et al. introduced the concept of the weighted pseudo almost automorphic function [9], which generalized the one of the weighted pseudo almost periodicity proposed in [10]. At present, there are many literatures to study the weighted pseudo almost automorphic function in other types of dynamics. For example, Chang et al. in [11] obtained the weighted pseudo almost automorphic solution for fractional differential equations. Besides, the weighted pseudo almost automorphic solution for stochastic partial differential equations has been discussed in $[12,13]$.

However, as far as we know, no author has studied the weighted pseudo almost automorphic solution for the nonautonomous difference equation as follows:

$$
u(k+1)=A(k) u(k)+f(k), \quad k \in \mathbb{Z},
$$

where $A(k)$ is specified later. Now, few authors investigated the properties of the solution to system (1). In 2013, Lizama and Mesquita have obtained almost automorphic solutions of (1); see [14]. But they did not deeply study the weighted pseudo almost automorphic solution of (1). This paper mainly discusses this problem and generalizes the result in [14].

The plan of the paper is as follows. In Section 2, some necessary definitions and results are stated. In Section 3, we discuss the weighted pseudo almost automorphic solutions of the linear nonautonomous difference equation. In Section 4, we obtain the weighted pseudo almost automorphic solutions of semilinear nonautonomous difference equation.

\section{Preliminaries}

In this section, we present some basic concepts and results. Let $X$ be any Banach space with the norm $\|\cdot\|$. BS $(\mathbb{Z}, X)$ is the space of all bounded sequences from $\mathbb{Z}$ to $X$ with the sup norm. To begin this paper, we recall some primary definitions of almost automorphy.

\section{Definition 1. Consider}

(i) Let $X$ be a (real or complex) Banach space. A function $u: \mathbb{Z} \rightarrow X$ is said to be discrete almost automorphic if for every integer sequence $\left(k_{n}^{\prime}\right)$ there exists a subsequence $\left(k_{n}\right)$ such that

$$
\lim _{n \rightarrow \infty} u\left(k+k_{n}\right)=\bar{u}(k)
$$


is well defined for each $k \in \mathbb{Z}$ and

$$
\lim _{n \rightarrow \infty} \bar{u}\left(k-k_{n}\right)=u(k)
$$

for each $k \in \mathbb{Z}$.

(ii) A function $f: \mathbb{Z} \times X \rightarrow X$ is said to be discrete almost automorphic if $f(k, x)$ is almost automorphic in $k \in \mathbb{Z}$ for any $x$.

Denote by $\mathrm{AA}_{d}(\mathbb{Z}, X)$ (resp., $\left.\mathrm{AA}_{d}(\mathbb{Z} \times X, X)\right)$ the set of all discrete almost automorphic function $f: \mathbb{Z} \rightarrow X$, (resp., $f: \mathbb{Z} \times X \rightarrow X)$. With the sup norm $\sup _{k \in \mathbb{Z}}\|u(k)\|$, this space is Banach spaces.

Theorem 2 (see [14]). If $u, v: \mathbb{Z} \times X \rightarrow X$ are discrete almost automorphic functions in $k$ for each $x$ in $X$, then the following assertions are true:

(1) $u+v$ is discrete almost automorphic in $k$ for each $x$ in $X$.

(2) $c u$ is discrete almost automorphic in $k$ for each $x$ in $X$, where $c$ is an arbitrary scalar.

(3) $\sup _{k \in \mathbb{Z}}\|u(k, x)\|=M_{x}<\infty$ for each $x$ in $X$.

(4) $\sup _{k \in \mathbb{Z}}\|\bar{u}(k, x)\|=N_{x}<\infty$, for each $x$ in $X$.

The following result can be found in [15, Theorem 2.10].

Theorem 3. Let $u: \mathbb{Z} \times X \rightarrow X$ be discrete almost automorphic in $k$ for each $x$ in $X$, satisfying a Lipschitz condition in $x$ uniformly in $k$; that is,

$$
\|u(k, x)-u(k, y)\| \leq L\|x-y\|, \quad \forall x, y \in X .
$$

Suppose $\varphi: \mathbb{Z} \rightarrow X$ is discrete almost automorphic; then the function $U: \mathbb{Z} \rightarrow X$ defined by $U(k)=u(k, \varphi(k))$ is discrete almost automorphic.

Let $\mathcal{U}$ be the set of all functions $\rho: \mathbb{Z} \rightarrow(0, \infty)$ which are positive and locally summable over $\mathbb{Z}$. For a given $K \in \mathbb{Z}_{+}$, set

$$
m_{d}(K, \rho):=\sum_{-K}^{K} \rho(k)
$$

for each $\rho \in \mathscr{U}$. Define

$$
\begin{aligned}
& \mathcal{U}_{\infty}:=\left\{\rho \in \mathcal{U}: \lim _{K \rightarrow \infty} m_{d}(K, \rho)=\infty\right\}, \\
& \mathcal{U}_{b}:=\left\{\rho \in \mathcal{U}_{\infty}: \rho \text { is bounded and } \inf _{k \in \mathbb{Z}} \rho(k)>0\right\} .
\end{aligned}
$$

Obviously, $\mathscr{U}_{b} \subset \mathscr{U}_{\infty} \subset \mathcal{U}$. Now for $\rho \in \mathscr{U}_{\infty}$ define

$$
\begin{aligned}
& \mathrm{BS}_{d}^{0}(\mathbb{Z}, \rho):=\{f \\
& \left.\quad \in \mathrm{BS}(\mathbb{Z}, X): \lim _{k \rightarrow \infty} \frac{1}{m_{d}(K, \rho)} \sum_{-K}^{K}\|f(k)\| \rho(k)=0\right\} .
\end{aligned}
$$

Similarly, we define $\mathrm{BS}_{d}^{0}(\mathbb{Z} \times X, \rho)$ as the collection of all functions $f: \mathbb{Z} \times X \rightarrow X$ is bounded and $f(\cdot, x)$ is bounded for each $x \in X$, and

$$
\lim _{K \rightarrow+\infty} \frac{1}{m_{d}(K, \rho)} \sum_{k=-K}^{K}\|f(k, x)\| \rho(k)=0
$$

uniformly in $x \in X$.

Definition 4. A bounded-sequence set $\mathscr{D}$ is said to be translation invariant if, for any $f(k) \in \mathscr{D}, f(k+\tau) \in \mathscr{D}$ for $k, \tau \in \mathbb{Z}$.

For simplicity, denote $\mathcal{U}^{\text {inv }}=\left\{\rho \in \mathcal{U}_{\infty}\right.$ : $\mathrm{BS}_{d}^{0}(\mathbb{Z}, \rho)$ is translation invariant $\}$. We are now ready to introduce the spaces $\mathrm{WPAA}_{d}(\mathbb{Z}, \rho)$ and $\mathrm{WPAA}_{d}(\mathbb{Z} \times X, \rho)$ of discrete weighted pseudo almost automorphic functions:

$$
\begin{aligned}
& \operatorname{WPAA}_{d}(\mathbb{Z}, \rho)=\{f=g+\phi \in \mathrm{BS}(\mathbb{Z}, X): g \\
& \left.\quad \in \mathrm{AA}_{d}(\mathbb{Z}, X), \phi \in \mathrm{BS}_{d}^{0}(\mathbb{Z}, \rho)\right\}, \\
& \mathrm{WPAA}_{d}(\mathbb{Z} \times X, \rho)=\{f=g+\phi \in \mathrm{BS}(\mathbb{Z} \times X, X): g \\
& \left.\quad \in \mathrm{AA}_{d}(\mathbb{Z} \times X, X), \phi \in \mathrm{BS}_{d}^{0}(\mathbb{Z} \times X, \rho)\right\} .
\end{aligned}
$$

Lemma 5. Assume that $g: \mathbb{Z} \rightarrow X$ is an almost automorphic function. Fix $k_{0} \in \mathbb{Z}, \in>0$, and write

$$
B_{\epsilon}:=\left\{\tau \in \mathbb{Z}:\left\|g\left(k_{0}+\tau\right)-g\left(k_{0}\right)\right\|<\epsilon\right\} .
$$

Then, there exist $k_{1}, \ldots, k_{m} \in \mathbb{Z}$ such that

$$
\bigcup_{i=1}^{m}\left(k_{i}+B_{\epsilon}\right)=\mathbb{Z}
$$

Proof. It is analogous to Lemma 2.1.1 in [16].

Lemma 6. If $f=g+\phi$ with $g \in A A_{d}(\mathbb{Z}, X)$ and $\phi \in$

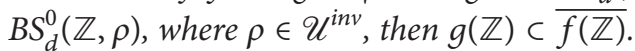

Proof. If this is not true, then there are $k_{0} \in \mathbb{Z}, \epsilon>0$ such that

$$
\left\|g\left(k_{0}\right)-f(k)\right\| \geq 2 \epsilon, \quad k \in \mathbb{Z} .
$$

Let $k_{1}, \ldots, k_{m}$ be as in Lemma 5 and write

$$
\begin{aligned}
\widehat{k}_{i} & =k_{i}-k_{0} \quad(1 \leq i \leq m), \\
\eta & =\max _{1 \leq i \leq m}\left|\widehat{k}_{i}\right| .
\end{aligned}
$$

For $k^{*} \in \mathbb{Z}_{+}$with $k^{*}>\eta$, we put

$$
\begin{aligned}
B_{\epsilon, k^{*}}^{(i)}=\left[-k^{*}+\eta-\widehat{k}_{i}, k^{*}-\eta-\widehat{k}_{i}\right] \cap\left(k_{0}+B_{\epsilon}\right), & \\
1 & \leq i \leq m,
\end{aligned}
$$

where $B_{\epsilon}$ is defined in Lemma 5. Using Lemma 5 gives that

$$
\bigcup_{i=1}^{m}\left(\widehat{k}_{i}+\eta+B_{\epsilon, k^{*}}^{(i)}\right)=\left[-k^{*}, k^{*}\right] \cap \mathbb{Z} .
$$


For any $k \in k_{0}+B_{\epsilon}$,

$$
\begin{aligned}
\|\phi(k)\| & =\|f(k)-g(k)\| \\
& \geq\left\|g\left(k_{0}\right)-f(k)\right\|-\left\|g(k)-g\left(k_{0}\right)\right\|>\epsilon .
\end{aligned}
$$

Thus, one yields

$$
\frac{1}{m_{d}\left(k^{*}, \rho\right)} \sum_{-k^{*}}^{k^{*}}\|\phi(k)\| \rho(k) \geq m \epsilon \longrightarrow m \epsilon
$$

as $k^{*} \longrightarrow \infty$.

This is a contradiction and this proof is completed.

Theorem 7. The decomposition of a discrete weighted pseudo almost automorphic function defined on $\mathbb{Z}$ is unique for any $\rho \in \mathcal{U}^{i n v}$.

Proof. Assume that $f=g_{1}+\phi_{1}$ and $f=g_{2}+\phi_{2}$ and then $0=\left(g_{1}-g_{2}\right)+\left(\phi_{1}-\phi_{2}\right)$. Since $g_{1}-g_{2} \in \mathrm{AA}_{d}(\mathbb{Z}, X)$ and $\phi_{1}-\phi_{2} \in \mathrm{BS}_{d}^{0}(\mathbb{Z}, \rho)$, we deduce that $g_{1}-g_{2}=0$ by Lemma 6 . Consequently, $\phi_{1}-\phi_{2}=0$; that is, $\phi_{1}=\phi_{2}$, which proves the uniqueness of the decomposition of $f$.

Theorem 8. If $\rho \in \mathcal{U}_{\infty}$, then $\left(W P A A_{d}(\mathbb{Z}, \rho),\|\cdot\|_{\infty}\right)$ is a Banach space.

Proof. Assume that $\left(f_{n}\right)$ is a Cauchy sequence in $\mathrm{WPAA}_{d}(\mathbb{Z}, \rho)$. We can write uniquely $f_{n}=g_{n}+\phi_{n}$. In view of Lemma 6 , we see that $\left\|g_{p}-g_{q}\right\|_{\infty} \leq\left\|f_{p}-f_{q}\right\|_{\infty}$, from which we deduce that $\left(g_{n}\right)$ is a Cauchy sequence in the Banach space $\mathrm{AA}_{d}(\mathbb{Z}, X)$. So, $\phi_{n}=f_{n}-g_{n}$ is also a Cauchy sequence in the Banach space $\mathrm{BS}_{d}^{0}(\mathbb{Z}, \rho)$. We can deduce that $g_{n} \rightarrow g \in \mathrm{AA}_{d}(\mathbb{Z}, X), \phi_{n} \rightarrow \phi \in \mathrm{BS}_{d}^{0}(\mathbb{Z}, \rho)$, and finally $f_{n} \rightarrow g+\phi \in \mathrm{WPAA}_{d}(\mathbb{Z}, \rho)$.

Lemma 9. Let $\phi \in B S(\mathbb{Z}, X)$. Then $\phi \in B S_{d}^{0}(\mathbb{Z}, \rho)$, where $\rho \in$ $\mathcal{U}_{\infty}$ if and only if, for any $\epsilon>0$,

$$
\lim _{K \rightarrow \infty} \frac{1}{m_{d}(K, \rho)} \sum_{k \in M_{K, \epsilon}(\phi)} \rho(k)=0
$$

where

$$
M_{K, \epsilon}(\phi)=\{k \in \mathbb{Z} \text { in }[-K, K] \mid\|\phi(k)\| \geq \epsilon\}
$$

Proof.

Necessity. For any $\epsilon>0$, we have

$$
\begin{aligned}
& \frac{1}{m_{d}(K, \rho)} \sum_{k=-K}^{K}\|\phi(k)\| \rho(k) \\
& =\frac{1}{m_{d}(K, \rho)}\left[\sum_{k \in M_{K, \epsilon}(\phi)}\|\phi(k)\| \rho(k)\right. \\
& \left.+\sum_{\substack{k \in \mathbb{Z}, k \in[-K, K] \backslash M_{K, \epsilon}(\phi)}}\|\phi(k)\| \rho(k)\right] \geq \frac{\epsilon}{m_{d}(K, \rho)}
\end{aligned}
$$

$$
\text { . } \sum_{k \in M_{K, \epsilon}(\phi)} \rho(k) \text {. }
$$

Noticing $\phi \in \mathrm{BS}_{d}^{0}(\mathbb{Z}, \rho)$, we thus know that

$$
\lim _{k \rightarrow+\infty} \frac{1}{m_{d}(k, \rho)} \sum_{k=-K}^{K}\|\phi(k)\| \rho(k)=0,
$$

which implies

$$
\lim _{K \rightarrow+\infty} \sum_{k \in M_{K, \epsilon}(\phi)} \rho(k)=0 .
$$

Sufficiency. From $\phi \in \mathrm{BS}_{d}^{0}(\mathbb{Z}, X)$, we know there exists a number $M>0$ such that $\|\phi(k)\| \leq M$ for all $k \in \mathbb{Z}$. Since $\lim _{K \rightarrow+\infty}\left(1 / m_{d}(K, \rho)\right) \sum_{k \in M_{K, \epsilon}(\phi)} \rho(k)=0$, for any $\epsilon>0$ there exists a number $K_{0} \in \mathbb{Z}_{+}$such that, for $K>K_{0}$,

$$
\frac{1}{m_{d}(K, \rho)} \sum_{k \in M_{K, \epsilon}(\phi)} \rho(k)<\frac{\epsilon}{M+1} .
$$

This gives

$$
\begin{aligned}
& \frac{1}{m_{d}(K, \rho)} \sum_{k=-K}^{K}\|\phi(k)\| \rho(k) \\
& =\frac{1}{m_{d}(K, \rho)}\left[\sum_{k \in M_{K, \epsilon}(\phi)}\|\phi(k)\| \rho(k)\right. \\
& \left.+\sum_{k \in[-K, K] \backslash M_{K, \epsilon}(\phi)}\|\phi(k)\| \rho(k)\right] \leq \frac{M}{m_{d}(K, \rho)} \\
& \sum_{k \in M_{K, \epsilon}(\phi)}^{\rho(k)+\frac{\epsilon}{m_{d}(K, \rho)}} \\
& \cdot \sum_{k \in[-K, K] \backslash M_{K, \epsilon}(\phi)}\|\phi(k)\| \rho(k) \leq \frac{M \epsilon}{M+1}+\epsilon<2 \epsilon .
\end{aligned}
$$

Thus $\phi \in \mathrm{BS}_{d}^{0}(\mathbb{Z}, \rho)$. This proof is finished. 
Theorem 10. Suppose $\rho \in \mathcal{U}^{i n v}, f(k, x) \in W P A A_{d}(\mathbb{Z} \times X, \rho)$, and there exists a number $L>0$ such that, for any $x, y \in X$,

$$
\|f(k, x)-f(k, y)\| \leq L\|x-y\|, \quad k \in \mathbb{Z} .
$$

Then, for any $x \in W P A A_{d}(\mathbb{Z}, \rho)$, one has $f(\cdot, x(\cdot)) \in$ $W P A A_{d}(\mathbb{Z}, \rho)$.

Proof. Since $f(k, x) \in \mathrm{WPAA}_{d}(\mathbb{Z} \times X, \rho)$, there exist two functions $g(k, x) \in \mathrm{AA}_{d}(\mathbb{Z} \times X, X)$ and $\phi(\mathbb{Z}, x) \in \mathrm{BS}_{d}^{0}(\mathbb{Z} \times$ $X, \rho)$ such that $f=g+\phi$. Also, since $x \in \mathrm{WPAA}_{d}(\mathbb{Z}, \rho)$, there exist $\alpha \in \mathrm{AA}_{d}(\mathbb{Z}, X)$ and $\beta \in \mathrm{BS}_{d}^{0}(\mathbb{Z}, \rho)$ such that $x=\alpha+\beta$. Hence,

$$
\begin{aligned}
f(k, x(k))= & g(k, \alpha(k))+f(k, x(k))-g(k, \alpha(k)) \\
= & g(k, \alpha(k))+f(k, x(k))-f(k, \alpha(k)) \\
& +\phi(k, \alpha(k)) .
\end{aligned}
$$

Let $G(k)=g(k, \alpha(k))$ and $W(k)=f(k, x(k))-f(k, \alpha(k))+$ $\phi(k, \alpha(k))$. Now, we are in a position to prove $G \in \mathrm{AA}_{d}(\mathbb{Z}, X)$ and $W \in \mathrm{BS}_{d}^{0}(\mathbb{Z}, \rho)$.

First, for any $x, y \in X$,

$$
\begin{aligned}
f(k, x)-f(k, y)= & g(k, x)+\phi(k, x)-g(k, y) \\
& -\phi(k, y) \\
= & g(k, x)-g(k, y)+\phi(k, x) \\
& -\phi(k, y) .
\end{aligned}
$$

Since $f(k, x)-f(k, y) \in \mathrm{WPAA}_{d}(\mathbb{Z}, \rho)$, from Lemma 6 , it follows that

$$
\begin{aligned}
& \{g(k, x)-g(k, x) \mid k \in \mathbb{Z}\} \\
& \quad \subset\{\overline{f(k, x)-f(k, y) \mid k \in \mathbb{Z}\},}
\end{aligned}
$$

so that

$$
\|g(\cdot, x)-g(\cdot, y)\|_{\infty} \leq\|f(\cdot, x)-f(\cdot, y)\|_{\infty} .
$$

Thus,

$$
\begin{aligned}
\|g(k, x)-g(k, y)\| & \leq\|g(\cdot, x)-g(\cdot, y)\|_{\infty} \\
& \leq L\|x-y\|
\end{aligned}
$$

for all $k \in \mathbb{Z}$. In view of $\alpha \in \mathrm{AA}_{d}(\mathbb{Z}, X)$ and from Theorem 3, it therefore follows that $G(k)=g(k, \alpha(k)) \in \mathrm{AA}_{d}(\mathbb{Z}, X)$.

Second, in order to prove $W \in \mathrm{BS}_{d}^{0}(\mathbb{Z}, \rho)$, we only need to prove $f(k, x(k))-f(k, \alpha(k)) \in \mathrm{BS}_{d}^{0}(\mathbb{Z}, \rho)$, and $\phi(k, \alpha(k)) \in$ $\mathrm{BS}_{d}^{0}(\mathbb{Z}, \rho)$. In fact, according to

$$
\begin{aligned}
& \|f(k, x(k))-f(k, \alpha(k))\| \leq L\|x(k)-\alpha(k)\| \\
& \quad \leq L\|\beta(k)\|
\end{aligned}
$$

and $\beta(k) \in \mathrm{BS}_{d}^{0}(\mathbb{Z}, X)$, we have

$$
\begin{gathered}
\frac{1}{m_{d}(K, \rho)} \sum_{k=-K}^{K}\|f(k, x(k))-f(k, \alpha(k))\| \rho(k) \\
\leq \frac{L}{m_{d}(K, \rho)} \sum_{k=-K}^{K}\|\beta(k)\|^{2} \rho(k) \longrightarrow 0,
\end{gathered}
$$

as $K \longrightarrow+\infty$.

Thus, $f(k, x(k))-f(k, \alpha(k)) \in \mathrm{BS}_{d}^{0}(\mathbb{Z}, \rho)$.

Since $\alpha: \mathbb{Z} \rightarrow X$, the set $\{\alpha(k)\}$ for $k \in \mathbb{Z}, k \in[-K, K]$ and some $K \in \mathbb{Z}_{+}$are finite. Its elements are denoted by $x_{1}, x_{2}, \ldots, x_{2 K}$. Hence, we have

$$
\begin{aligned}
& \{k \in \mathbb{Z} \mid k \in[-K, K],\|\phi(k, \alpha(k))\| \geq \epsilon\} \\
& \quad \subset \bigcup_{i=1}^{2 K}\left\{k \in \mathbb{Z} \mid k \in[-K, K],\left\|\phi\left(k, x_{i}\right)\right\| \geq \epsilon\right\} .
\end{aligned}
$$

Further, we have

$$
\begin{aligned}
& \frac{1}{m_{d}(K, \rho)} \sum_{k \in M_{K, \epsilon}(\phi(k, \alpha(k)))} \rho(k) \\
& \leq \sum_{i=1}^{2 K} \frac{1}{m_{d}(K, \rho)} \sum_{k \in M_{K, \epsilon}\left(\phi\left(k, x_{i}\right)\right)} \rho(k) .
\end{aligned}
$$

Notice $\phi\left(k, x_{i}\right) \in \mathrm{BS}_{d}^{0}(\mathbb{Z} \times X, \rho)$. Then, by virtue of Lemma 9, we obtain

$$
\lim _{K \rightarrow+\infty} \frac{1}{m_{d}(K, \rho)} \sum_{k \in M_{K, \epsilon}\left(\phi\left(k, x_{i}\right)\right)} \rho(k)=0
$$

for $i=1,2, \ldots, 2 K$, which consequently gives

$$
\lim _{K \rightarrow+\infty} \frac{1}{m_{d}(K, \rho)} \sum_{k \in M_{K, \epsilon}(\phi(k, \alpha(k)))} \rho(k)=0 .
$$

Therefore, according to Lemma 9 again, we know $\phi(k, \alpha(k)) \epsilon$ $\mathrm{BS}_{d}^{0}(\mathbb{Z}, \rho)$. From the above discussion, we obtain that $f(k, x(k)) \in \mathrm{WPAA}_{d}(\mathbb{Z}, \rho)$, and this completes proof.

\section{Weighted Pseudo Almost Automorphic Solutions of Nonautonomous Line Difference Equation}

In this section, consider the following nonautonomous linear difference equation:

$$
u(k+1)=A(k) u(k)+f(k), \quad k \in \mathbb{Z},
$$

where $A(k)$ are given nonsingular $n \times n$ matrices with elements $a_{i j}(k), 1 \leq i, j \leq n, f: \mathbb{Z} \rightarrow E^{n}$ is given $n \times 1$ vector function, and $u(k)$ is an unknown $n \times 1$ vector with components $u_{i}(k), 1 \leq i \leq n$. Its associated homogeneous linear difference equation is given by

$$
u(k+1)=A(k) u(k), \quad k \in \mathbb{Z} .
$$


Definition 11. The matrix $U\left(k, k_{0}\right)$ which satisfies (38) and $U\left(k_{0}, k_{0}\right)=1$ is called principal fundamental matrix. One will denote $U\left(k, k_{0}\right)$ by $U(k) U^{-1}\left(k_{0}\right)$.

From [14], the following holds:

$$
\begin{aligned}
U(k) & U^{-1}\left(k_{0}\right) \\
= & \begin{cases}\prod_{l=k_{0}}^{k-1} A\left(k_{0}+k-1-l\right), & \forall k-0 \leq k \in \mathbb{Z}, \\
\prod_{l=k}^{k_{0}-1} A^{-1}(l), & \forall k_{0} \geq k \in \mathbb{Z} .\end{cases}
\end{aligned}
$$

Definition 12 (see [14]). A matrix function $A(k)$ is said to be discrete almost automorphic if each entry of the matrix is discrete almost automorphic function; that is,

$$
\lim _{n \rightarrow \infty} A\left(k+k_{n}\right)=: \bar{A}(k)
$$

is well defined for each $k \in \mathbb{Z}$ and

$$
\lim _{n \rightarrow \infty} \bar{A}\left(k-k_{n}\right)=A(k)
$$

for each $k \in \mathbb{Z}$.

Lemma 13 (see [14]). Suppose $A(k)$ is discrete almost automorphic and a nonsingular matrix on $\mathbb{Z}$. Also, suppose that the set $\left\{A^{-1}(k)\right\}_{k \in \mathbb{Z}}$ is bounded. Then $A^{-1}(k)$ is almost automorphic on $\mathbb{Z}$; that is, for every sequence of integer numbers $\left(k_{n}^{\prime}\right)$, there exists a subsequence $\left(k_{n}\right)$ such that

$$
\lim _{n \rightarrow \infty} A^{-1}\left(k+k_{n}\right)=: \bar{A}^{-1}(k)
$$

is well defined for each $k \in \mathbb{Z}$ and

$$
\lim _{n \rightarrow \infty} \bar{A}^{-1}\left(k-k_{n}\right)=A^{-1}(k)
$$

for each $k \in \mathbb{Z}$.

Definition 14. Let $U(k)$ be the principal fundamental matrix of (38). System (38) is said to possess an exponential dichotomy if there exist a projection $P$, which commutes with $U(k)$, and positive constants $\eta, \nu, \alpha$, and $\beta$ such that, for all $k, l \in \mathbb{Z}$, one has

$$
\begin{aligned}
\left\|U(k) P U^{-1}(l)\right\| & \leq \eta e^{-\alpha(k-l)}, \quad k \geq l, \\
\left\|U(k)(I-P) U^{-1}(l)\right\| \leq v e^{-\beta(l-k)}, & l \geq k .
\end{aligned}
$$

Specially, for $\alpha=\beta=0$, system (44) is said to possess an ordinary dichotomy.

According to Theorem 2.12 in [14], if system (38) possesses an exponential dichotomy and the function $f: \mathbb{Z} \rightarrow$ $E^{n}$ is bounded, then system (37) has a bounded solution which is given by

$$
\begin{aligned}
u(k)= & \sum_{j=-\infty}^{k-1} U(k) P U^{-1}(j+1) f(j) \\
& -\sum_{j=k}^{\infty} U(k)(I-P) U^{-1}(j+1) f(j),
\end{aligned}
$$

We start by proving a result concerning existence of a discrete weighted pseudo almost automorphic solution of (37).

Theorem 15. Suppose $A(k)$ is discrete almost automorphic and nonsingular matrix and the set $\left\{A^{-1}(k)\right\}_{k \in \mathbb{Z}}$ is bounded. Also, suppose the function $f \in W P A A_{d}(\mathbb{Z}, \rho)$, where $\rho \in \mathcal{U}^{i n v}$, and (38) admits an exponential dichotomy with positive constants $\nu, \eta, \beta$, and $\alpha$. Then system (37) has a discrete weighted pseudo almost automorphic solution.

Proof. Since $f: \mathbb{Z} \rightarrow E^{n}$ is a discrete weighted pseudo almost automorphic function, we have $f=g+\phi$, where $g \in$ $\mathrm{AA}_{d}\left(\mathbb{Z}, E^{n}\right)$ and $\phi \in \mathrm{BS}_{d}^{0}(\mathbb{Z}, \rho)$, which is unique according to Theorem 7. In the help of (45), the solution of the system (37) is

$$
\begin{aligned}
u(k)= & \sum_{j=-\infty}^{k-1} U(k) P U^{-1}(j+1)[g(j)+\phi(j)] \\
& -\sum_{j=k}^{\infty} U(k)(I-P) U^{-1}(j+1)[g(j)+\phi(j)] \\
= & \sum_{j=-\infty}^{k-1} U(k) P U^{-1}(j+1) g(j) \\
& -\sum_{j=k}^{\infty} U(k)(I-P) U^{-1}(j+1) g(j) \\
& +\sum_{j=-\infty}^{k-1} U(k) P U^{-1}(j+1) \phi(j) \\
& -\sum_{j=k}^{\infty} U(k)(I-P) U^{-1}(j+1) \phi(j) .
\end{aligned}
$$

Let

$$
\begin{aligned}
\Phi(k)= & \sum_{j=-\infty}^{k-1} U(k) P U^{-1}(j+1) g(j) \\
& -\sum_{j=k}^{\infty} U(k)(I-P) U^{-1}(j+1) g(j), \\
\Psi(k)= & \sum_{j=-\infty}^{k-1} U(k) P U^{-1}(j+1) \phi(j) \\
& -\sum_{j=k}^{\infty} U(k)(I-P) U^{-1}(j+1) \phi(j) .
\end{aligned}
$$

By using proof method of Theorem 3.1 in [14], one can know that $\Phi$ in (47) is almost automorphic on $\mathbb{Z}$. Thus, we only need to prove that $\Psi \in \mathrm{BS}_{d}^{0}(\mathbb{Z}, \rho)$. Next, we pass to prove

$$
\lim _{K \rightarrow \infty} \frac{1}{m_{d}(K, \rho)} \sum_{k=-K}^{K}\|\Psi(k)\| \rho(k)=0 .
$$


In fact, we have

$$
\begin{aligned}
& \frac{1}{m_{d}(K, \rho)} \sum_{k=-K}^{K}\|\Psi(k)\| \rho(k) \leq \frac{1}{m_{d}(K, \rho)} \\
& \cdot \sum_{k=-K}^{K}\left\|\sum_{j=-\infty}^{k-1} U(k) P U^{-1}(j+1) \phi(j)\right\| \rho(k) \\
& +\frac{1}{m_{d}(K, \rho)} \\
& \cdot \sum_{-K}^{K}\left\|\sum_{j=k}^{\infty} U(k)(I-P) U^{-1}(j+1) \phi(j)\right\| \rho(k) \\
& \leq \frac{1}{m_{d}(K, \rho)} \\
& \cdot \sum_{k=-K}^{K} \sum_{j=-\infty}^{k-1}\left\|U(k) P U^{-1}(j+1) \phi(j)\right\| \rho(k) \\
& +\frac{1}{m_{d}(K, \rho)} \\
& \cdot \sum_{k=-K}^{K} \sum_{j=k}^{\infty}\left\|U(k)(I-P) U^{-1}(j+1) \phi(j)\right\| \rho(k) \\
& \leq \frac{\eta}{m_{d}(K, \rho)} \sum_{k=-K}^{K} \sum_{j=-\infty}^{k-1} e^{-\alpha(k-j-1)}\|\phi(j)\| \rho(k) \\
& +\frac{v}{m_{d}(K, \rho)} \sum_{k=-K}^{K} \sum_{j=k}^{\infty} e^{-\beta(j+1-k)}\|\phi(j)\| \rho(k) \\
& =\frac{\eta}{m_{d}(K, \rho)} \sum_{k=-K}^{K} \sum_{j=0}^{\infty} e^{-\alpha j}\|\phi(k-j-1)\| \rho(k) \\
& +\frac{v}{m_{d}(K, \rho)} \sum_{k=-K}^{K} \sum_{j=1}^{\infty} e^{-\beta j}\|\phi(j+k-1)\| \rho(k) .
\end{aligned}
$$

From the well-known Tonelli theorem, it follows that

$$
\begin{aligned}
& \frac{1}{m_{d}(K, \rho)} \sum_{k=-K}^{K} \sum_{j=0}^{\infty} e^{-\alpha j}\|\phi(k-j-1)\| \rho(k) \\
& =\sum_{j=0}^{\infty} e^{-\alpha j}\left[\frac{1}{m_{d}(K, \rho)} \sum_{k=-K}^{K}\|\phi(k-j-1)\| \rho(k)\right] \\
& \frac{1}{m_{d}(K, \rho)} \sum_{k=-K}^{K} \sum_{j=1}^{\infty} e^{-\beta j}\|\phi(j+k-1)\| \rho(k) \\
& =\sum_{j=1}^{\infty} e^{-\beta j}\left[\frac{1}{m_{d}(K, \rho)} \sum_{k=-K}^{K}\|\phi(j+k-1)\| \rho(k)\right] .
\end{aligned}
$$

Since $\rho \in \mathcal{U}^{\text {inv }}$ and $\phi \in \mathrm{BS}_{d}^{0}(\mathbb{Z}, \rho)$, we can know that, for any $j \in \mathbb{Z}$,

$$
\begin{aligned}
& \frac{1}{m_{d}(K, \rho)} \sum_{k=-K}^{K}\|\phi(k-j-1)\| \rho(k) \longrightarrow 0, \\
& \frac{1}{m_{d}(K, \rho)} \sum_{k=-K}^{K}\|\phi(j+k-1)\| \rho(k) \longrightarrow 0,
\end{aligned}
$$

as $K \rightarrow+\infty$. Define two functions as follows:

$$
\begin{aligned}
& f_{1}^{(K)}(x)
\end{aligned}
$$

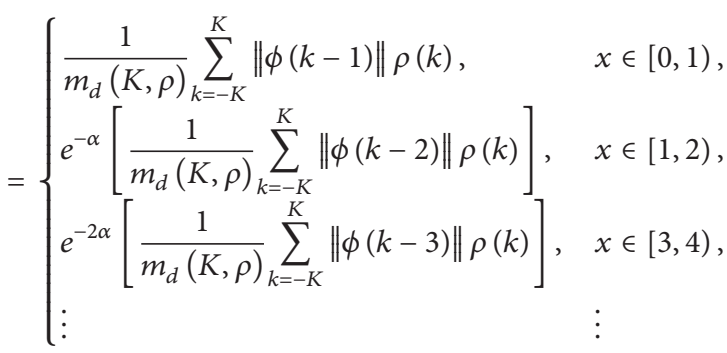

$$
\begin{aligned}
& f_{2}^{(K)}(x, K)
\end{aligned}
$$

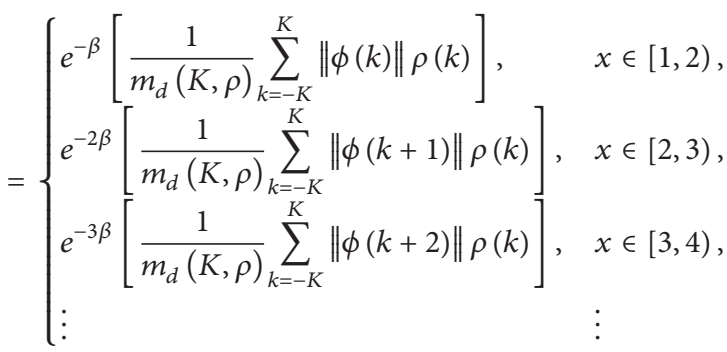

for any fixed $K \in \mathbb{Z}_{+}$. Obviously, the sequences $f_{1}^{(K)}, f_{2}^{(K)}$ are measurable on $\mathbb{R}$ and

$$
\begin{aligned}
& \sum_{j=0}^{\infty} e^{-\alpha j}\left[\frac{1}{m_{d}(K, \rho)} \sum_{k=-K}^{K}\|\phi(k-j-1)\| \rho(k)\right] \\
& =\int_{0}^{+\infty} f_{1}^{(K)}(x) d x, \\
& \sum_{j=1}^{\infty} e^{-\beta j}\left[\frac{1}{m_{d}(K, \rho)} \sum_{k=-K}^{K}\|\phi(j+k-1)\| \rho(k)\right] \\
& =\int_{1}^{+\infty} f_{2}^{(K)}(x) d x .
\end{aligned}
$$

Besides, in view of (52), one knows that

$$
\begin{aligned}
& \lim _{K \rightarrow+\infty} f_{1}^{(K)} \longrightarrow 0, \\
& \lim _{K \rightarrow+\infty} f_{2}^{(K)} \longrightarrow 0 .
\end{aligned}
$$


Then by the Lebesgue dominated convergence theorem, we have

$$
\begin{aligned}
& \lim _{K \rightarrow+\infty} \frac{1}{m_{d}(K, \rho)} \sum_{k=-K}^{K} \sum_{j=0}^{\infty} e^{-\alpha j}\|\phi(k-j-1)\| \rho(k) \\
& =\lim _{K \rightarrow+\infty} \sum_{j=0}^{\infty} e^{-\alpha j}\left[\frac{1}{m_{d}(K, \rho)} \sum_{k=-K}^{K}\|\phi(k-j-1)\|\right. \\
& \cdot \rho(k)]=\lim _{K \rightarrow+\infty} \int_{0}^{+\infty} f_{1}^{(K)}(x) d x=0, \\
& \lim _{K \rightarrow+\infty} \frac{1}{m_{d}(K, \rho)} \sum_{k=-K}^{K} \sum_{j=1}^{\infty} e^{-\beta j}\|\phi(j+k-1)\| \\
& =\lim _{K \rightarrow+\infty} \sum_{j=1}^{\infty} e^{-\beta j}\left[\frac{1}{m_{d}(K, \rho)}\right. \\
& \left.\cdot \sum_{k=-K}^{K}\|\phi(j+k-1)\| \rho(k)\right] \\
& =\lim _{K \rightarrow+\infty} \int_{1}^{+\infty} f_{2}^{(K)}(x) d x=0 .
\end{aligned}
$$

Thus, one obtains that

$$
\lim _{K \rightarrow \infty} \frac{1}{m_{d}(K, \rho)} \sum_{k=-K}^{K}\|\Psi(k)\| \rho(k)=0,
$$

and this proof is completed.

\section{Weighted Pseudo Almost Automorphic Solutions for Semilinear Nonautonomous Difference Equation}

In this section, we consider the following difference equation:

$$
u(k+1)=A(k) u(k)+f(k, u(k)), \quad k \in \mathbb{Z},
$$

where $A(k)$ is described as in (37); besides $f: \mathbb{Z} \times E^{n} \rightarrow E^{n}$ is a given $n \times 1$ vector function.

Definition 16. One says that $u: \mathbb{Z} \rightarrow E^{n}$ is solution of (58), if it satisfies

$$
\begin{aligned}
u(k)= & \sum_{j=-\infty}^{k-1} U(k) P U^{-1}(j+1) f(j, u(j)) \\
& -\sum_{j=k}^{\infty} U(k)(I-P) U^{-1}(j+1) f(j, u(j)),
\end{aligned}
$$

for every $k \in \mathbb{Z}$.

Now, the following result shows the existence and uniqueness of a weighted pseudo almost automorphic solution of (58).
Theorem 17. Suppose $A(k)$ is discrete almost automorphic and nonsingular matrix and the set $\left\{A^{-1}(k)\right\}_{k \in \mathbb{Z}}$ is bounded. Also, assume that (38) admits an exponential dichotomy with positive constants $\nu, \eta, \beta$, and $\alpha$. Assume the function $f \in$ $W P A A_{d}\left(\mathbb{Z} \times E^{n}, \rho\right)$, satisfying the following condition: there exists a constant $0<L<\left(\eta /\left(1-e^{-\alpha}\right)+\nu e^{-\beta} /\left(1-e^{-\beta}\right)\right)^{-1}$ such that

$$
\|f(k, u)-f(k, v)\| \leq L\|u-v\|
$$

for every $u, v \in E^{n}$, and $k \in \mathbb{Z}$. Then system (58) has a discrete weighted pseudo almost automorphic solution.

Proof. Since the function $f$ satisfies the global Lipschitztype condition, we obtain by Theorem 10 that $f(\cdot, u(\cdot))$ is in $\mathrm{WPAA}_{d}(\mathbb{Z}, \rho)$ for any $u \in \mathrm{WPAA}_{d}(\mathbb{Z}, \rho)$. Define an operator $T$ as follows:

$$
\begin{aligned}
(T u)(k):= & \sum_{j=-\infty}^{k-1} U(k) P U^{-1}(j+1) f(j, u(j)) \\
& -\sum_{j=k}^{\infty} U(k)(I-P) U^{-1}(j+1) f(j, u(j))
\end{aligned}
$$

for all $u \in \mathrm{WPAA}_{d}(\mathbb{Z}, \rho)$ and $U$ is the fundamental matrix of (38). By Theorem 15, one can know the operator $T$ : $\mathrm{WPAA}_{d}(\mathbb{Z}, \rho) \rightarrow \mathrm{WPAA}_{d}(\mathbb{Z}, \rho)$. Thus we only need to prove that the operator $T$ is contraction in $\mathrm{WPAA}_{d}(\mathbb{Z}, \rho)$. In fact,

$$
\begin{aligned}
\|T u-T v\|_{\infty} \leq & \eta L \sum_{j=-\infty}^{k-1} e^{-\alpha(k-j-1)}\|u-v\|_{\infty} \\
& +\nu L \sum_{j=k}^{+\infty} e^{-\beta(j+1-k)}\|u-v\|_{\infty} \\
\leq & \eta L \sum_{j=0}^{+\infty} e^{-\alpha j}\|u-v\|_{\infty} \\
& +v L \sum_{j=1}^{+\infty} e^{-\beta j}\|u-v\|_{\infty} \\
\leq & L\left(\frac{\eta}{1-e^{-\alpha}}+\frac{v e^{-\beta}}{1-e^{-\beta}}\right)\|u-v\|_{\infty} .
\end{aligned}
$$

Since $L<\left(\eta /\left(1-e^{-\alpha}\right)+\nu e^{-\beta} /\left(1-e^{-\beta}\right)\right)^{-1}$, we obtain that $T$ is a contraction. Then, $T$ has a unique fixed point. Thus, system (58) has a unique solution which is discrete weighted pseudo almost automorphic one.

\section{Competing Interests}

The authors declare that they have no competing interests.

\section{Acknowledgments}

This work is supported by Grants 11563005 and 61563033 from the National Natural Science Foundation of China and 20151BAB212011 and 20151BAB201021 from the Natural Science Foundation of Jiangxi Province. 


\section{References}

[1] S. Bochner, "Uniform convergence of monotone sequences of functions," Proceedings of the National Academy of Sciences of the United States of America, vol. 47, pp. 582-585, 1961.

[2] J. Liu, G. M. N’Guérékata, and N. V. Minh, "Almost automorphic solutions of second order evolution equations," Applicable Analysis, vol. 84, no. 11, pp. 1173-1184, 2005.

[3] C. Cuevas and C. Lizama, "Almost automorphic solutions to a class of semilinear fractional differential equations," Applied Mathematics Letters, vol. 21, no. 12, pp. 1315-1319, 2008.

[4] J. P. C. D. Santos and C. Cuevas, "Asymptotically almost automorphic solutions of abstract fractional integro-differential neutral equations," Applied Mathematics Letters, vol. 23, no. 9, pp. 960-965, 2010.

[5] M. Adıvar and H. C. Koyuncuoğlu, "Almost automorphic solutions of discrete delayed neutral system," Journal of Mathematical Analysis and Applications, vol. 435, no. 1, pp. 532-550, 2016.

[6] G. M. N'Guérékata, Topics in Almost Automorphy, Springer Science \& Business Media, 2007.

[7] J. Shao, "Pseudo almost periodic solutions for a LasotaWazewska model with an oscillating death rate," Applied Mathematics Letters, vol. 43, pp. 90-95, 2015.

[8] C. Y. Zhang, "Pseudo-almost-periodic solutions of some differential equations," Journal of Mathematical Analysis and Applications, vol. 181, no. 1, pp. 62-76, 1994.

[9] J. Blot, G. M. Mophou, G. M. N’Guérékata, and D. Pennequin, "Weighted pseudo almost automorphic functions and applications to abstract differential equations," Nonlinear Analysis, Theory, Methods \& Applications, vol. 71, no. 3-4, pp. 903-909, 2009.

[10] R. P. Agarwal, B. De Andrade, and C. Cuevas, "Weighted pseudo-almost periodic solutions of a class of semilinear fractional differential equations," Nonlinear Analysis: Real World Applications, vol. 11, no. 5, pp. 3532-3554, 2010.

[11] Y.-K. Chang, R. Zhang, and G. M. N’Guérékata, "Weighted pseudo almost automorphic mild solutions to semilinear fractional differential equations," Computers and Mathematics with Applications, vol. 64, no. 10, pp. 3160-3170, 2012.

[12] Z. Chen and W. Lin, "Square-mean weighted pseudo almost automorphic solutions for non-autonomous stochastic evolution equations," Journal de Mathmatiques Pures et Appliques, vol. 100, no. 4, pp. 476-504, 2013.

[13] K. Li, "Weighted pseudo almost automorphic solutions for nonautonomous SPDEs driven by Lévy noise," Journal of Mathematical Analysis and Applications, vol. 427, no. 2, pp. 686721, 2015.

[14] C. Lizama and J. G. Mesquita, "Almost automorphic solutions of non-autonomous difference equations," Journal of Mathematical Analysis and Applications, vol. 407, no. 2, pp. 339-349, 2013.

[15] D. Araya, R. Castro, and C. Lizama, "Almost automorphic solutions of difference equations," Advances in Difference Equations, vol. 2009, Article ID 591380, 15 pages, 2009.

[16] W. A. Veech, "Almost automorphic functions on groups," American Journal of Mathematics, vol. 87, pp. 719-751, 1965. 


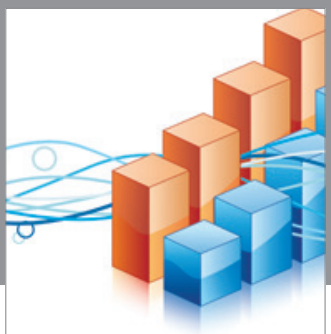

Advances in

Operations Research

vatem alat4

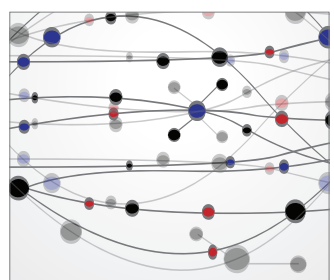

\section{The Scientific} World Journal
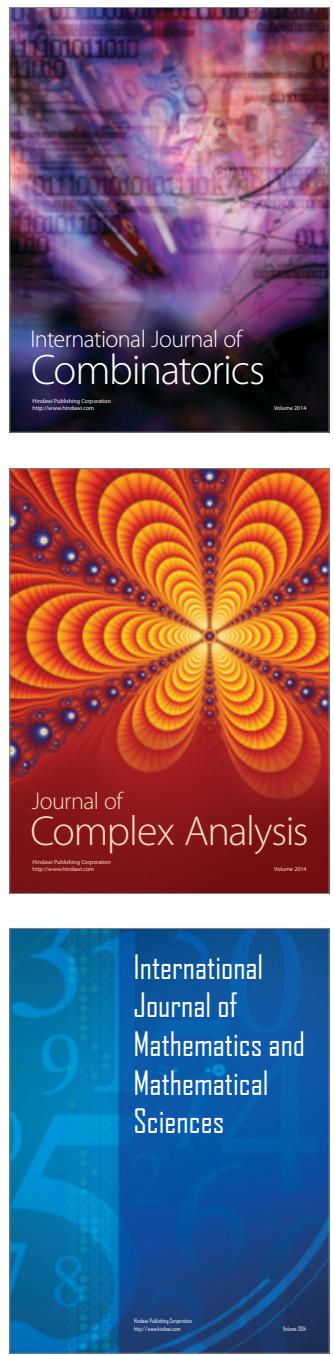
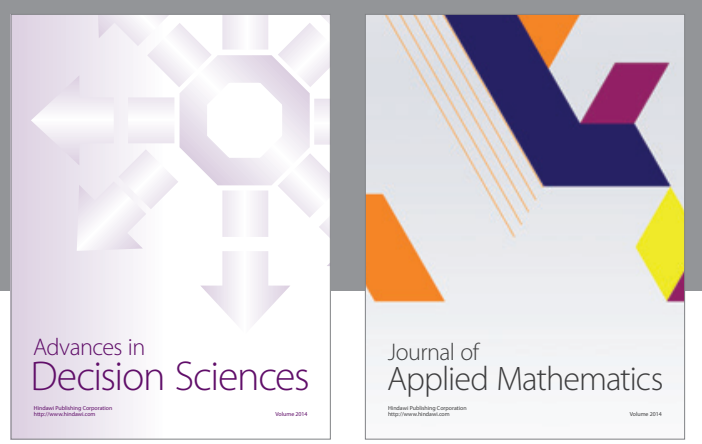

Algebra

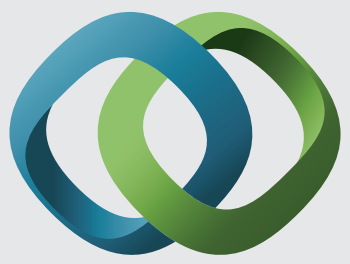

\section{Hindawi}

Submit your manuscripts at

http://www.hindawi.com
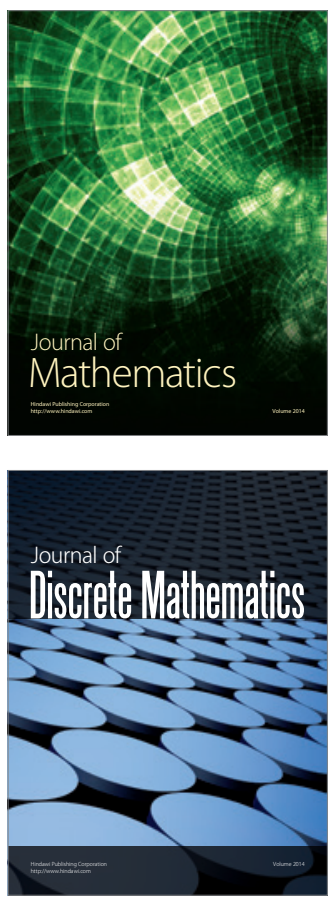

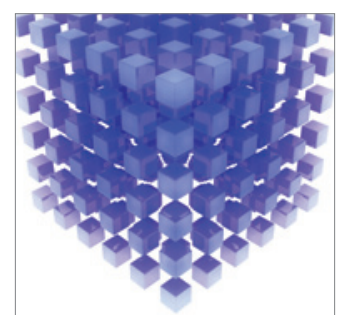

Mathematical Problems in Engineering
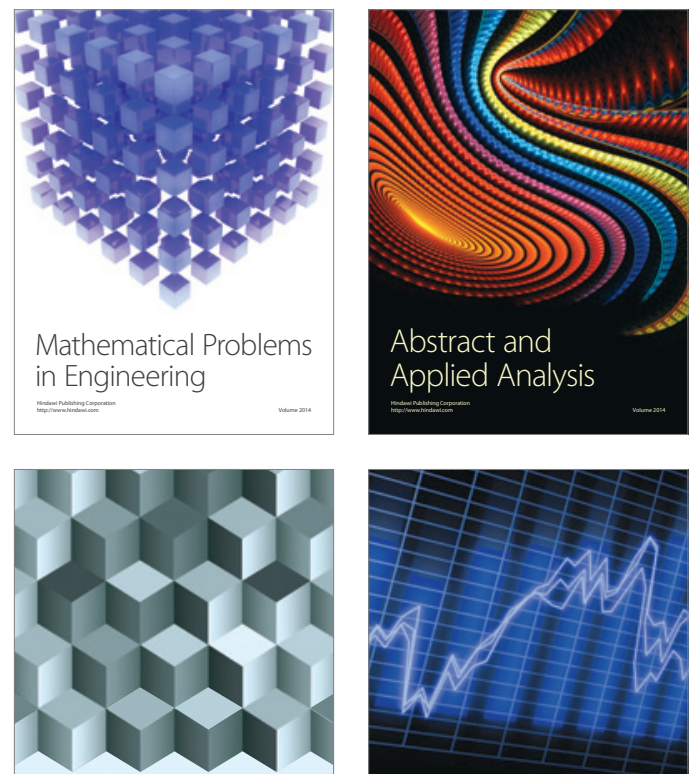

Journal of

Function Spaces

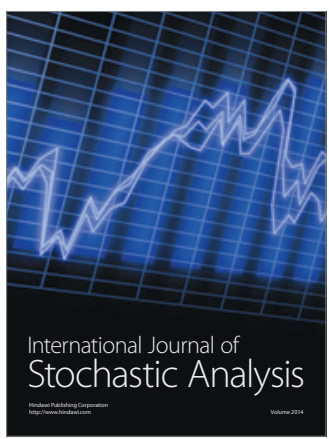

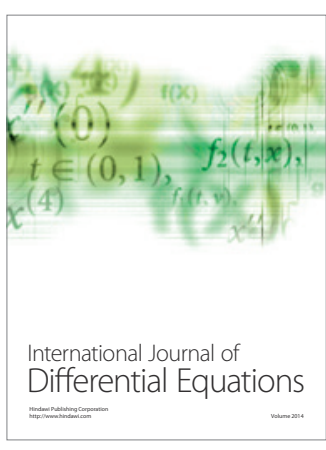
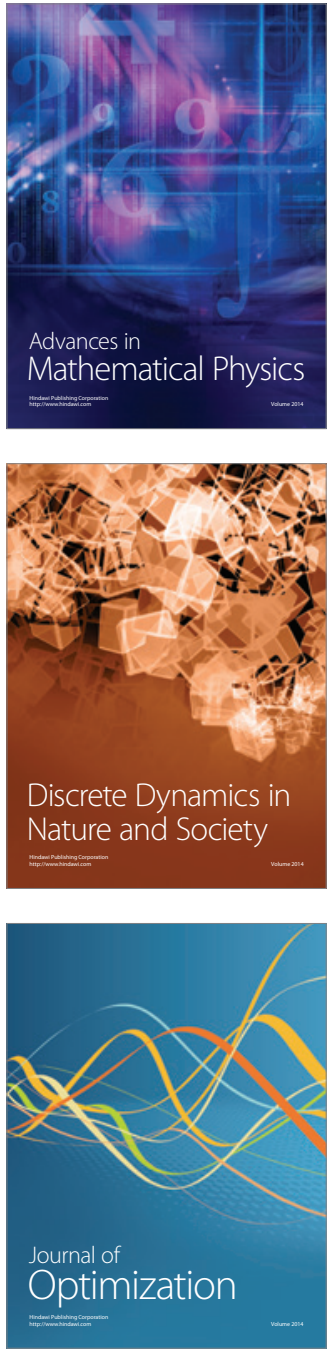